\title{
Evoked Potential Detection using Circular Shift Invariant Discrete Wavelet Transform
}

\author{
Bhargav N. ${ }^{1}$, Dr. Viswanatha V.M. ${ }^{2}$, Dr. Shailesh M.L. ${ }^{3}$ \\ ${ }^{1}$ Research Scholar, Dept. of ECE, HKE's SLNCE, Raichur, Karnataka, India, bhargavn1@gmail.com \\ ${ }^{2}$ HOD, Dept. of ECE, HKE's SLN College of Engineering, Raichur, Karnataka, India, \\ vmviswanatha@gmail.com \\ ${ }^{3}$ Professor, Dept. of BME, RGIT, Bengaluru, Karnataka, India, shailesh.ml@gmail.com
}

\begin{abstract}
Signal processing uses mathematical analysis and measurements to distinguish data from various sensor types that is concealed in signal. Ambiguity and artifacts defile the biomedical signals. The ideal signal is not readily available or identifiable in various applications. The problem of signal evaluation is to retrieve the ideal signal from a debased copy in the most optimal possible way. Once the EEG is obtained, the Wavelet Transform Ratio for calculation exceeds the SNR (Signal Noise ratio) by a massive amount in a single sweep. This adds potential from scalp electrons, background events and other disturbances to the EEG. Within this paper, the two separate transform wavelet systems, Circular shift Invariant and Translation Invariant Wavelet Transform techniques were addressed to boost the SNR.
\end{abstract}

Key words : Circular Shift Invariant, Translation Invariant, Visual Evoked Potential, Ensemble Averaging, Discrete Wavelet Transform (DWT), and SNR

\section{INTRODUCTION}

In this excellent world, the brain is the most confounding structure. The brain controls a number of components, each of which has extraordinary specific functionalities. Evoked potential (EP) is called the brain electrical movement which takes place with regard to foreign impetus. In case of subjective actions, whenever the perceptual analysis is conducted, the response signal is frequently referred to as the event-related potential (ERP)[4]. EPs are valuable indicative instruments when contemplating the physiological and psychological conditions of the subject under consideration.

The EP (signal) and the continuous EEG (noise) in the estimates are the focus of this paper[8]. For this reason, It requires use of incredible techniques such as Bio-Medical Digital Signal Processing and plethora of other procedures [7].

\subsection{Visual Evoked Potential (VEP)}

Evoked potential (EP) develops a latest generally clinical neurophysiology technology which allows neural system functional evaluation. These non-obtrusive techniques provide data on valuable conditions inside the nervous system of different expansions[1]. In recognition of subclinical brokenness, evoked potential are particularly valuable. The primary Visual Evoked Potential (VEP) recognition reflects EEG disclosure. It has been found that when an amplified light stimulus is applied, electrical activity in the brain is affected. The use of this approach was high when computerized averaging techniques were implemented and because of the very lower power.

\subsection{Earlier Methods}

It can usually be shown that the signal is misaligned by noise during analysis of real biomedical signals. Arithmetic mean is one of the modes used for low-pass filtering noise attenuation. The traditional band-pass filters are extremely unambiguous, but often very inefficient, as signal frequency and noise overlap considerably.

The weight of each sample is adjusted in the weighted average method, considering a statistic parameter, a standard deviation[5]. For the study, two EP samples were taken into account. Each averaging signal is given weights in this procedure before averaging to achieve a weighted average brain potentials and these weights optimise the resulting average SNR if certain conditions are fulfilled.

The adaptive noise cancelation for Evoked Potentials with adaptive filtering is met with in this context. Analysis of a wavelet: a variable size windowing technique. By using Wavelet Analysis [6] we can obtain more precise low frequency data at long interludes and larger recurrence data from smaller areas.

It is well known that the traditional Wavelet Transform is responsive to the position of a signal and that the energy distribution of two-signal wavelet coefficients can vary 
Bhargav N. et al., International Journal of Emerging Trends in Engineering Research, 8(9), September 2020, 6363 - 6368

considerably even though the two signals are only different by time (or space). This inconvenience can cause serious issues in using the multiresolution wavelet display for tasks such as pattern discrimination or recognition.

The notion of invariance of translation (or invariance of shifts) in literature most of the time (or space) involves finding the best set of Wavelet Transform coefficients for representing the signal. The standard discrete wavele transform is an ideal system for data compression, because the wavelet coefficients are very small in redundancies and correlations.

However, it is very important that a good representation of a signal is not prone to shift and scaling when dealing with problems such as pattern discriminations and recognition

\section{Methodologies}

\subsection{Ensemble Averaging Technique}

The Ensemble Averaging method is the most frequent way to estimate evoked potential. Ensemble Averaging is the production and integration of several models for generating the necessary output by the averaging of subsequent data from the same data point for the processing of replicated signal data in place of a single model.

Signal averages are determined by the following signal and noise attributes.

- Repeating signals are taken into account

- Signal and random noise in nature must be unconnected.

- Precise knowledge of each signal sample's time position is important

SNR increases as the total number of trials is higher.

\section{Ensemble Averaging Technique Algorithm}

- Different memory sites hold the same ensemble data.

- Incorporate the primary assessments of the various exhibits and reserve them in the first position in different cluster; all place assessments must also be taken into account and stored - The average is determined by dividing by quantity of tests

- A Signal-to - Noise ratio plot is calculated by determining and positioning SNR in arrays for each sweep

\subsection{Wavelet Transform Technique}

Transforming Wavelet has raised a large enthusiasm in the area of signal processing[10]. They find applications in some regions such as voice-coding, edge recognition, information containment, deriving up of acknowledgment, research requirements, and so on, since wavelets are used as a method for information investigation and change in information at different resolution levels [2].

\section{Daubechies Wavelet Approximation}

Non-linear approximation leads to the thresholding of low potential wavelet coefficients [3]. The approximation of M-term is defined as

$$
\mathrm{fM}=\sum|\mathrm{f}, \psi \mathrm{j}, \mathrm{n}| \mid>\mathrm{T}(\mathrm{f}, \psi \mathrm{j}, \mathrm{n}) \psi \mathrm{j}, \mathrm{n} .
$$

\section{Biorthogonal wavelet}

The biorthogonal wavelet is a transition in the wavelet, where the wavelet's transformation is non-symmetric but invertible. Biorthogonal wavelets will attain greater degrees of freedom when compare with orthogonal wavelets. A further degree of freedom allows for the symmetrical wavelet functions.

There are two scaling functions in the case of Biorthogonal, which can produce many analyzes and two wavelet functions $\varphi, \varphi^{\prime}$ accordingly. The sequences of scaling $a, a^{\prime}$ can vary. Scaling sequences must satisfy the following biorthogonality requirements

$\sum_{n \in Z} a_{n} a_{n+2 m}^{\prime}=2 * \partial_{m, o}$

Then the wavelet Sequences may be specified as

$$
\begin{aligned}
& b_{n}=(-1)^{n^{\prime}} a_{M-1-n} \quad(\mathrm{n}=0,1, \ldots, ., \mathrm{N}-1) \\
& b_{n}^{\prime}=(-1)^{n} a_{M-1-n} \quad(\mathrm{n}=0,1, \ldots ., \mathrm{N}-1)
\end{aligned}
$$

\subsection{Algorithm for Wavelet Transform Technique}

- The signal decay by administering the transforming discrete wavelet as shown in fig 1

- Expel the high repetition signal e.g. the informative coefficients and keep the low recurrence signal e.g.

- Reproduce the Evoked Potential signal by using the disintegrated signal's reverse wavelet transform and as shown in Fig 2.

- Make every accurate coefficient to zero and transform using the reverse wavelet

- Finding the signal-to-noise ratio for various studies 
Bhargav N. et al., International Journal of Emerging Trends in Engineering Research, 8(9), September 2020, $6363-6368$

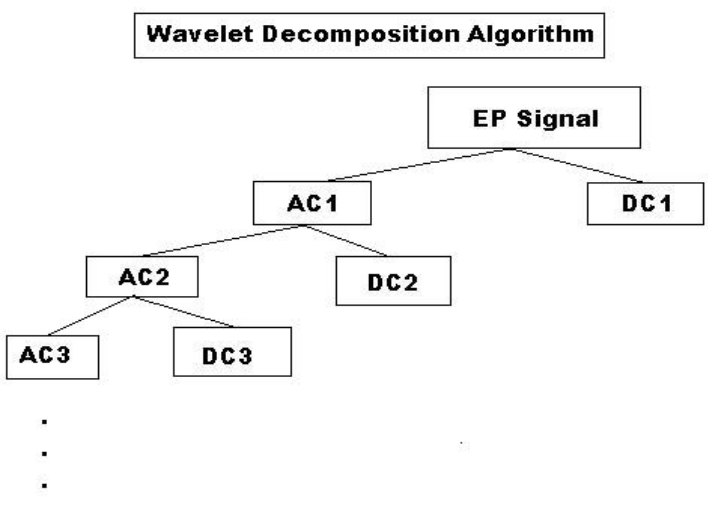

Figure 1: Decomposition of EP Signal

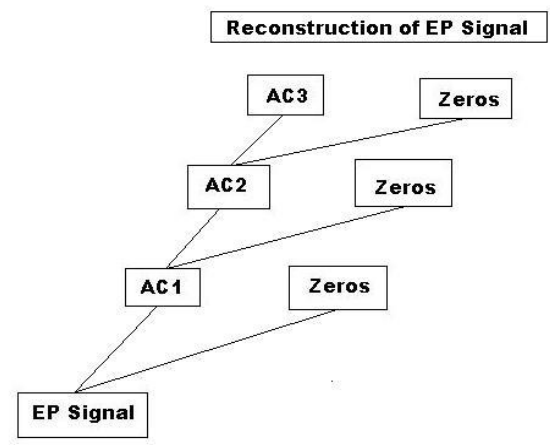

Figure 2: Reconstruction of EP Signal.

\subsection{Translation Invariant and Circular Shift Invariant Discrete Wavelet Transform Technique}

In typical non-invariant de-noising, the harsh oscillations in the region of discontinuities imply that it generates significantly greater oscillations than the soft threshold.

However, some of these oscillations might be damped by the translation-invariant approach. If used in a translation-invariant way at large sampling speeds, hard thresholds looks so fantastic that translation invariant (TI) methods can be considered at considerably smaller speeds than would usually be beneficial with a wavelet reduction. There are many ways to wavelet shrinkage, and the 2 $\log (\mathrm{n})^{3 / 4}$ version for different purposes can be strengthened. The universal threshold $(\sigma \sqrt{ } 2 \log (\mathrm{N}))$ intended to remove noise-driven spikes that compromise the smoothness of the reconstruction[11]. However, if you want to calculate output only by a mean squared error, then we have stronger lower thresholds. Unfortunately, these lower thresholds result in an immense amount of noise spikes when used in combination with translation-invariant noise, which are seemingly considerably greater than those in a non-invariant case[9].

In order to produce the Translation Invariant wavelet calculation for the Evoked Potential, the information must be shifted, the shifted data thresholded, the thresholds unshifted and then the sum of all shifts must be summed [4]. As the Translation Invariant wavelet is applied separately to each study, other experiments wont be influenced and is helpful in single-stimulus estimation of Evoked Potential.

The evaluation of the evoked potential can be described as a three-stage, wavelet decay, non-linear threshold, and inverting process. Thresholding is a key step in the isolation of the signal from the noise construct. The wavelet coefficients at different levels are sometimes little to no association with each level approximately white noise. Thus level-dependent thresholds are naturally followed. The threshold at the jth stage has the following form for the TI wavelet dependent Evoked Potential estimator:

$$
\mathrm{Th}_{\mathrm{J}}=\sigma \sqrt{2 \log _{\mathrm{e}}\left(N \log _{2}(N)\right)}
$$

where $\sigma=\mathrm{MAD}_{\mathrm{J}} / 0.6745, \mathrm{MAD}_{\mathrm{J}}$ is median absolute value estimated on the wavelet coefficients at the $\mathrm{j}^{\text {th }}$ level.

\section{Algorithm for Translation Invariant Method using Discrete Wavelet Transform}

- Take the one EP data epoch and store in an array

- Apply DWT on an initial EP data epoch

- Apply the soft threshold to the decomposed data and store

- Next, shift the EP data by one position to the right

- Apply the DWT for the shifted EP data

- Again, apply the soft threshold to the decomposed data and store

- Next, unshift the soft thresholded data

- Reconstruct the signal and store in separate array

- Repeat step 4 to step 8 for $\mathrm{N}$ number of shifts

- Average for all shifted data

- Plot the results

- Calculate the SNR

\section{Algorithm for Circular Shift Invariant Method using Discrete Wavelet Transform}

- Take the one EP data epoch and store in an array

- Apply DWT on an initial EP data epoch

- Apply the soft threshold to the decomposed data and store

- Next, shift the EP data circularly by one position

- Apply the DWT for the circularly shifted EP data

- Again, apply the soft threshold to the decomposed data and store

- Next, shift back circularly the soft thresholded data

- Reconstruct the signal and store in separate array

- Repeat step 4 to step 8 for $\mathrm{N}$ number of shifts

- Average for all shifted data

- Plot the results

- Calculate the SNR 
Bhargav N. et al., International Journal of Emerging Trends in Engineering Research, 8(9), September 2020, 6363 - 6368

\section{RESULTS}

Counterfeit data was taken into consideration here and is shown in Figure 3 showing three separate tests of information considered individually at Trial No.20, Trial number 40 and Trial Number 60. Every test of 300 inspects, for the investigation 60 similar trials of information were considered. Just three information trials appear in the figure below.
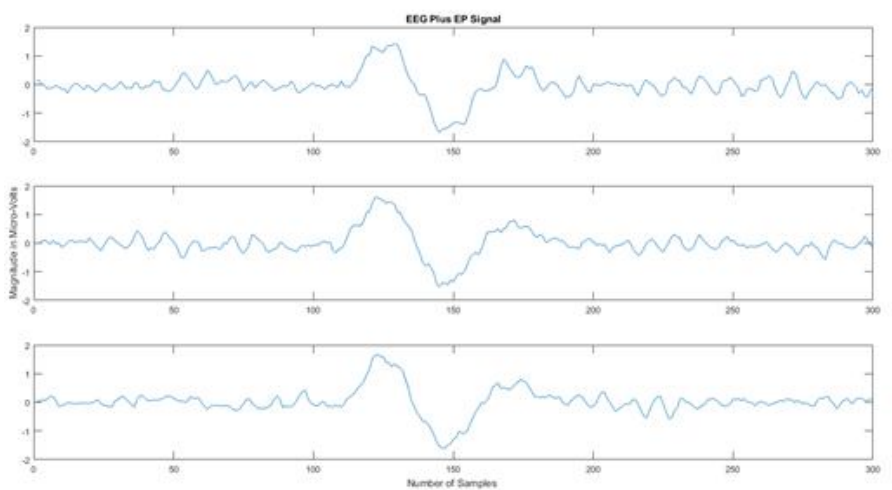

Figure 3: EEG plus EP Signal

In order to get the yield and to determine SNR values, the ensemble averaging procedure considers 60 information tests.

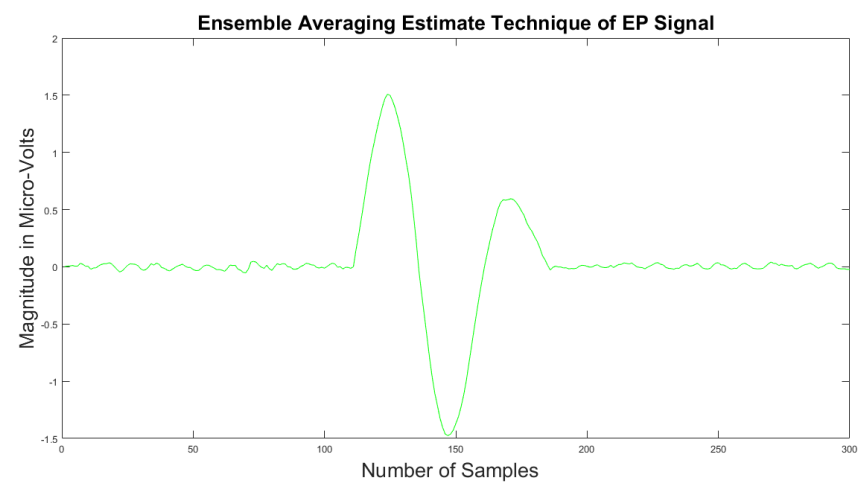

Figure 4: Ensemble Averaging Output Signal

Figure 4 shows the Ensemble averaging procedure's yield waveform. The figure shows the average number of reiterative signals to the EP signal function. Table 1 shows that SNR upgrades by a portion of a practically square base of a number of tests as the number of trials is progressively taken into consideration. The noise ratio signal (SNR), as shown in Figure 5, to the number of sweeps.

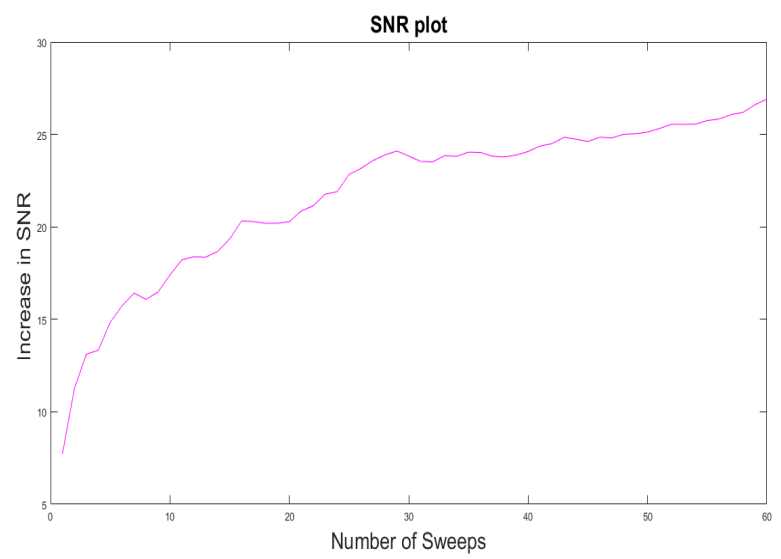

Figure 5: Signal to Noise Ratio (SNR) plot

Table 1: Ensemble Averaging Technique SNR for Various Trials

\begin{tabular}{|c|c|c|}
\hline Data & $\begin{array}{c}\text { Number } \\
\text { of Trials }\end{array}$ & $\begin{array}{c}\text { Ensemble Averaging } \\
\text { Technique SNR in dB }\end{array}$ \\
\hline 1 & 10 & $16.1 \mathrm{~dB}$ \\
\hline 2 & 20 & $17.73 \mathrm{~dB}$ \\
\hline 3 & 40 & $20.58 \mathrm{~dB}$ \\
\hline 4 & 60 & $22.78 \mathrm{~dB}$ \\
\hline
\end{tabular}

The Translation Invariant Wavelet Transform technique as compared with the original EP signal is as shown in figure 6.
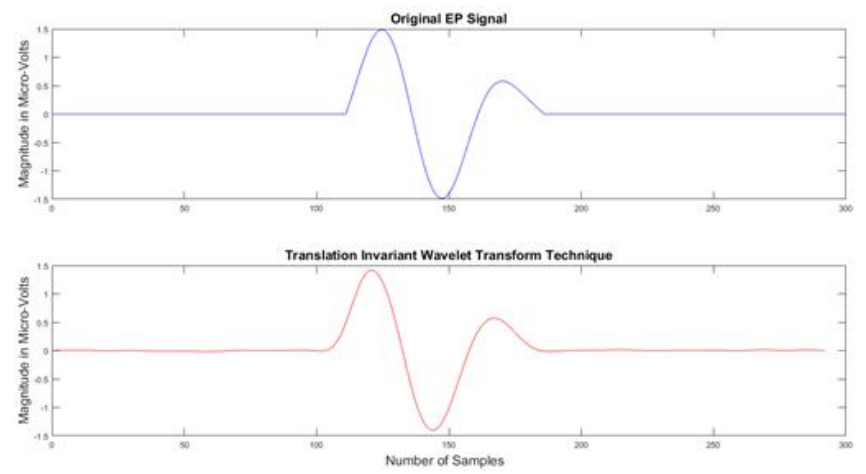

Figure 6: Original EP signal and Translation Invariant Wavelet Transform Technique

Comparison of Ensemble Averaging Technique with Translation Invariant Wavelet Transform technique was drawn up and shown in Figure 7. From the waveform, Translation Invariant Wavelet Transform technique provides stronger extract of the EP signal from the EEG background than Ensemble Averaging. 
Bhargav N. et al., International Journal of Emerging Trends in Engineering Research, 8(9), September 2020, 6363 - 6368
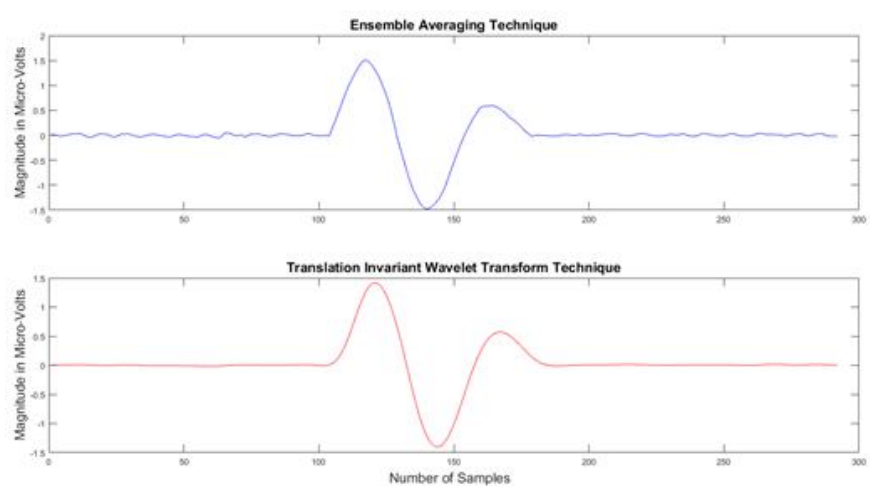

Figure 7: Ensemble Averaging Technique and Translation Invariant Wavelet Transform Technique

A plot of SNR values in $\mathrm{dB}$ for different shifts in Translation Invariant Wavelet Transform technique is as shown in figure 8.

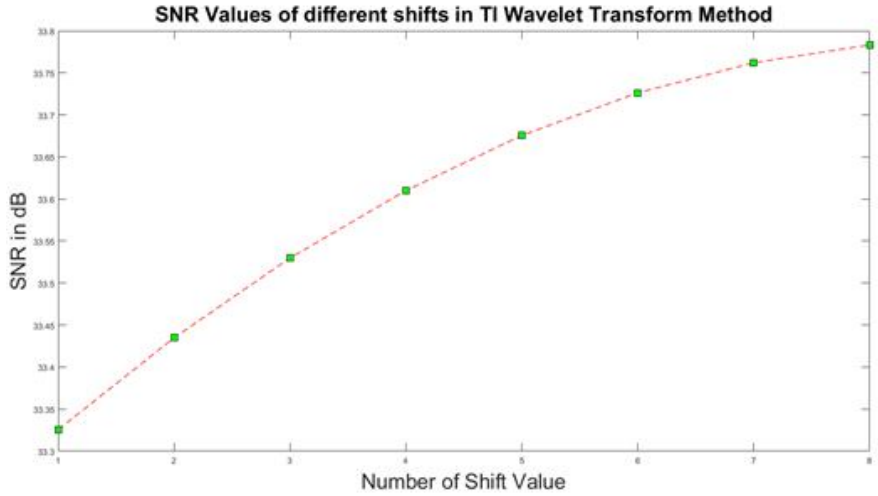

Figure 8: Output SNR's for different shifts in TIWT

SNR values in $\mathrm{dB}$ for Daubechies, Symlet and Bi-Orthogonal Wavelet Transform Translation Invariant (TI) techniques for different Trials have been tabulated in table 2 .

Table 2: SNR values of different Wavelet Transform Translation Invariant (TI) techniques for Various Trials

\begin{tabular}{|c|l|l|l|}
\hline $\begin{array}{l}\text { Shift } \\
\text { Values }\end{array}$ & $\begin{array}{l}\text { Daubechies } \\
\text { Wavelet } \\
\text { Transform TI } \\
\text { Method }\end{array}$ & $\begin{array}{l}\text { Symlet } \\
\text { Wavelet } \\
\text { Transform } \\
\text { TI Method }\end{array}$ & $\begin{array}{l}\text { Bi-Orthogonal } \\
\text { Wavelet Transform } \\
\text { TI Method }\end{array}$ \\
\hline 1 & 33.3255 & 33.3237 & 33.3135 \\
\hline 2 & 33.4349 & 33.4322 & 33.4233 \\
\hline 3 & 33.5298 & 33.5263 & 33.5190 \\
\hline 4 & 33.6101 & 33.6064 & 33.6000 \\
\hline 5 & 33.6756 & 33.6723 & 33.6659 \\
\hline 6 & 33.7263 & 33.7242 & 33.7162 \\
\hline 7 & 33.7621 & 33.7618 & 33.7507 \\
\hline 8 & 33.7832 & 33.7848 & 33.7698 \\
\hline
\end{tabular}

The Circular Shift Invariant Wavelet Transform technique as compared with the original EP signal is as shown in figure 9.
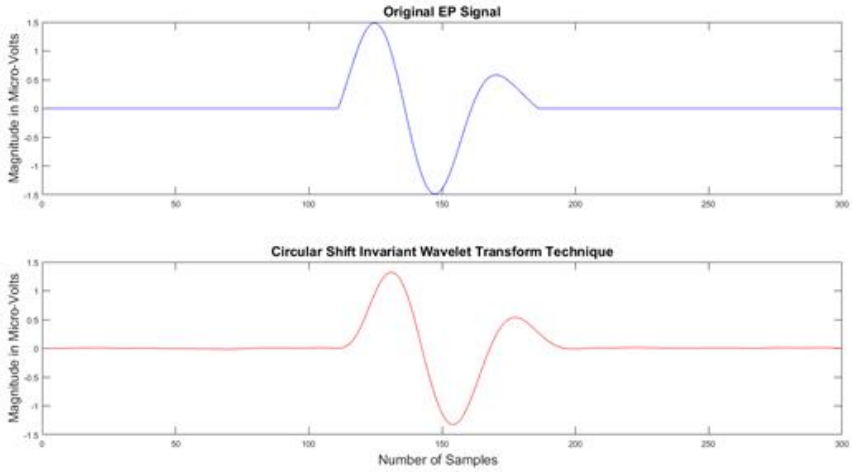

Figure 9: Original EP signal and Daubechies Wavelet Circular Shift Invariant Wavelet Transform Technique

Comparison of Ensemble Averaging Technique with Circular Shift Invariant Wavelet Transform technique was drawn up and shown in Figure 10. From the waveform, Circular Shift Invariant Wavelet Transform technique provides stronger extract of the EP signal from the EEG background than Ensemble Averaging.
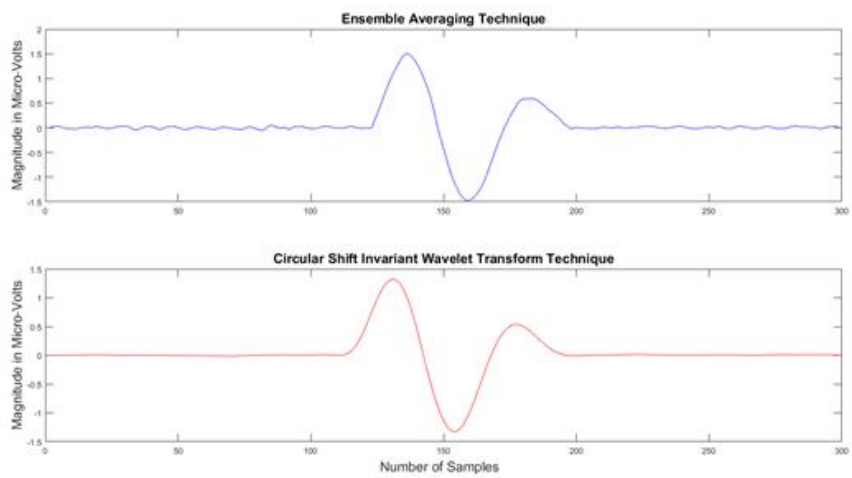

Figure 10: Ensemble Averaging Technique and Circular Shift Invariant Wavelet Transform Technique

A plot of SNR values in dB for different shifts in Circular Shift Invariant Wavelet Transform technique is as shown in figure 11.

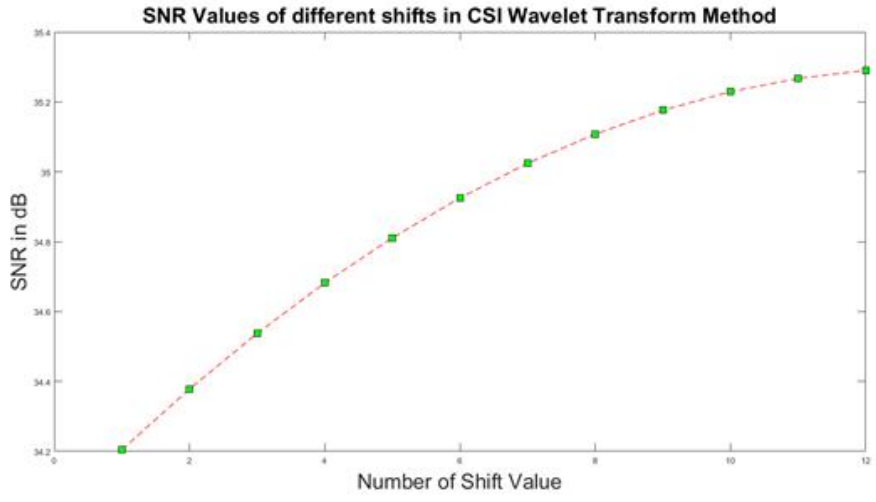

Figure 11: Output SNR's for different shifts in CSIWT 
Bhargav N. et al., International Journal of Emerging Trends in Engineering Research, 8(9), September 2020, $6363-6368$

SNR values in $\mathrm{dB}$ for Daubechies, Symlet and Bi-Orthogonal Wavelet Transform Circular Shift Invariant (CSI) techniques for different Trials has been tabulated in table 3 .

Table 3: SNR values of different Wavelet Transform Circular Shift Invariant (CSI) techniques for Various Trials

\begin{tabular}{|c|l|c|c|}
\hline $\begin{array}{l}\text { Shift } \\
\text { Values }\end{array}$ & $\begin{array}{l}\text { Daubechies } \\
\text { Wavelet } \\
\text { Transform CSI } \\
\text { Method }\end{array}$ & $\begin{array}{l}\text { Symlet } \\
\text { Wavelet } \\
\text { Transform } \\
\text { CSI Method }\end{array}$ & $\begin{array}{l}\text { Bi-Orthogonal } \\
\text { Wavelet Transform } \\
\text { CSI Method }\end{array}$ \\
\hline 1 & 34.2044 & 34.2040 & 34.1926 \\
\hline 2 & 34.3781 & 34.3773 & 34.3663 \\
\hline 3 & 34.5373 & 34.5359 & 34.5255 \\
\hline 4 & 34.6817 & 34.6798 & 34.6697 \\
\hline 5 & 34.8111 & 34.8092 & 34.7984 \\
\hline 6 & 34.9253 & 34.9240 & 34.9115 \\
\hline 7 & 35.0242 & 35.0240 & 35.0087 \\
\hline 8 & 35.1079 & 35.1091 & 35.0905 \\
\hline 9 & 35.1762 & 35.1790 & 35.1574 \\
\hline 10 & 35.2294 & 35.2335 & 35.2098 \\
\hline 11 & 35.2674 & 35.2723 & 35.2482 \\
\hline 12 & 35.2904 & 35.2952 & 35.2725 \\
\hline
\end{tabular}

\section{CONCLUSION}

Ensemble Averaging Technique has the downside of several thousand sweep numbers, which are then calculated on average for several minutes and cause unwarranted movement artifacts in some cases, including infant patients. It is therefore highly desirable to reduce the acquisition time of EP calculation.

The new method proposed i.e Translation Invariant and Circular Shift Invariant Discrete Wavelet Transform technique has been discussed here and it has been compared with Ensemble Averaging Technique using Daubechies Wavelet Transform, Symlet Wavelet Transform and Bi-Orthogonal Wavelet Transform. Circular Shift Invariant Wavelet based Techniques provides with better SNR when compared with its counterparts as shown in the results.

\section{REFERENCES}

1. Groseth GS, Ashman EJ. Practice parameter: the usefulness of evoked potentials in identifying clinically silent lesions in patients with suspected multiple sclerosis (an evidenced based review). Neurology2000; 54:1720-5. Report of the Quality

2. M P Wachowiak, G S Rash, P M Quesada, A H Desoky in IEEE Transactions on Biomedical Engineering (2000), Wavelet-based noise removal for biomechanical signals: a comparative study.

3. Jeena Joy, Salice Peter, Neetha John, "Denoising Using Soft Thresholdng", International Journal of Advanced
Research in Electrical, Electronics and Instrumentation Engineering Vol.2, Issue 3, March 2013

4. An Improved Invariant Wavelet Transform, XinYang Huanga*, MinSheng Tana, HongRu Wub, Lijun Ouyanga,Li Liua, International Workshop on Information and Electronics Engineering (IWIEE),2012

5. Laciar, E. \& Jane, R. (2001). An Improved Weighted Signal Averaging Method for High-Resolution ECG Signals, Computers in Cardiology, Vol. 28, pp. 69-72, ISSN:0276-6574.

6. A wavelet tour of signal processing, Stephane Mallat, PHI Publications

7. Monson H. Hayes: Statistical Digital Signal Processing and Modeling, Wiley, 1996, ISBN 0-471-59431-8

8. Willis.J.Tomkins Bio Medical Digital Signal Processing, PHI Publications

9. Bhargav N., Dr.Viswanatha V.M., Dr.Shailesh M.L., "Evoked Potential Detection using LMS Adaptive Wiener Filter and Wavelet Transform", International Journal of Recent Technology and Engineering, 2019

10. John Martin Ladrido, Jonnel Alejandrino, Emmanuel Trinidad and Lawrence Materum "Comparative Survey of Signal Processing and Artificial Intelligence Based Channel Equalization Techniques and Technologies", IJETER, volume 7, no 9, 2019

11. Jagadish S.Jakati, Shridhar S.Kuntoji, "Efficient Speech De-noising Algorithm using Multi-level Discrete Wavelet Transform and Thresholding", Volume 8 No. 6, 2020 